\title{
REABILITAR EM CONTEXTO DE PANDEMIA PELA COVID-19: UM RELATO DE EXPERIÊNCIA
}

\section{REHABILITATION IN THE CONTEXT OF A PANDEMIC BY COVID-19: AN EXPERIENCE REPORT}

\section{REHABILITACIÓN EN EL CONTEXTO DE UNA PANDEMIA POR COVID-19: UN INFORME DE EXPERIENCIA NURSING CARE IN}

DOI 10.33194/rper.2022.194

Data de receção: 2021-09-23 Data de aceitação: 2022-01-11 Data de publicação on-line: 2022-01-22

\author{
Maria Arminda Leonor ${ }^{1}$; $;$ Marlene Teixeira ${ }^{1}(\mathbb{D}) ;$ Silvia Pereira ${ }^{1}\left(\mathbb{D}\right.$; Olga Ribeiro ${ }^{2}(\mathbb{D}$ \\ 1 Centro Hospitalar Universitário de São João, Porto, Portugal; \\ 2 Escola Superior de Enfermagem do Porto; Centro de Investigação em Tecnologias e Serviços de Saúde, Porto, Portugal
}

Autor correspondente: Maria Arminda Leonor, mariaarmindaleonor@hotmail.com

\section{RESUMO}

Contextualização: 0 surgimento de uma nova doença na China, provocada por uma estirpe de coronavírus (SARSCoV-2), rapidamente teve impacto a nível Mundial, obrigando a uma reestruturação social e de saúde.

Objetivo: Relatar a experiência vivenciada pelos enfermeiros especialistas em enfermagem de reabilitação durante a prestação de cuidados especializados num serviço coorte de doentes com COVID-19.

Método: Relato de experiência, descritivo e retrospetivo, referente ao exercício profissional dos enfermeiros especialistas em enfermagem de reabilitação de um serviço coorte de doentes com COVID-19, num hospital do Norte de Portugal, no período de fevereiro de 2020 a julho de 2021.

Resultados: Desde o início da pandemia, foi exigido aos serviços de saúde e aos profissionais, uma capacidade de adaptação sem precedentes, no sentido de gerir com mais eficácia os cuidados a prestar aos doentes com COVID19. Numa fase inicial, os enfermeiros de reabilitação foram fundamentais na gestão da equipa e determinantes na conceção, implementação e reformulação dos cuidados, atuando sempre no sentido de promover a recuperação, a qualidade de vida e diminuir a incapacidade. Nos registos no SClínico, verificou-se que as atividades diagnósticas e intervenções mais frequentes estavam focadas na reabilitação respiratória, sem, no entanto, menosprezar a reabilitação motora.

Conclusão: 0 facto de algumas das intervenções especializadas não terem sido asseguradas diretamente, pode ter retardado a obtenção de ganhos em saúde. No entanto, apesar de todas as dificuldades, os enfermeiros especialistas em enfermagem de reabilitação tiveram um papel preponderante nas transições vivenciadas pelos doentes com COVID-19, sem nunca descurarem o envolvimento dos cuidadores.

DESCRITORES: Enfermagem em Reabilitação; Pandemia; Infeções por Coronavírus

\section{ABSTRACT}

Background: The emergence of a new disease in China, caused by a strain of coronavirus (SARS-CoV-2), quickly had an impact worldwide, forcing a social and health restructuring.

Objective: Report the experience of specialist nurses in rehabilitation nursing during the provision of specialized care in a cohort service of patients with COVID-19.

Method: Experience report, descriptive and retrospective, referring to the professional practice of specialist nurses in rehabilitation nursing in a cohort service of patients with COVID-19, in a hospital in Northern Portugal, from February 2020 to July 2021.

Results: Since the beginning of the pandemic, health services and professionals were required to have an unprecedented capacity to adapt, in order to manage more effectively the care provided to patients with COVID19. Initially, rehabilitation nurses were fundamental in managing the team and determining the design, implementation and reformulation of care, always working to promote recovery, quality of life and reduce disability. In the records in the Clinic, it was found that the most frequent diagnostic activities and interventions were focused on respiratory rehabilitation, without, however, underestimating motor rehabilitation.

Conclusion: The fact that some of the specialized interventions were not directly assured may have delayed the achievement of health gains. However, despite all the difficulties, specialist nurses in rehabilitation nursing played 
a major role in the transitions experienced by patients with COVID-19, without ever neglecting the involvement of caregivers

DESCRIPTORS: Rehabilitation Nursing; Pandemic; Coronavirus Infections

\section{RESUMEN}

Introducción: La aparición de una nueva enfermedad en China, causada por una cepa de coronavirus (SARS-CoV-2), tuvo rápidamente un impacto en todo el mundo, lo que obligó a una reestructuración social y sanitaria.

Objetivo: Informar sobre la experiencia de enfermeros especialistas en rehabilitación de enfermería durante la prestación de atención especializada en un servicio de cohorte de pacientes con COVID-19.

Método: Informe de experiencia, descriptivo y retrospectivo, referido a la práctica profesional de enfermeros especialistas en enfermería de rehabilitación en un servicio de cohorte de pacientes con COVID-19, en un hospital del norte de Portugal, desde febrero de 2020 hasta julio de 2021.

Resultados: Desde el inicio de la pandemia se requirió que los servicios y profesionales de salud tuvieran una capacidad de adaptación sin precedentes, con el fin de gestionar de manera más efectiva la atención brindada a los pacientes con COVID-19. Inicialmente, las enfermeras de rehabilitación fueron fundamentales en la gestión del equipo y en la determinación del diseño, implementación y reformulación de la atención, trabajando siempre para promover la recuperación, la calidad de vida y la reducción de la discapacidad. En los registros de la Clínica se constató que las actividades e intervenciones diagnósticas más frecuentes se centraron en la rehabilitación respiratoria, sin, sin embargo, subestimar la rehabilitación motora.

Conclusión: El hecho de que algunas de las intervenciones especializadas no estuvieran aseguradas directamente puede haber retrasado el logro de beneficios para la salud. Sin embargo, a pesar de todas las dificultades, los enfermeros especialistas en rehabilitación de enfermería jugaron un papel importante en las transiciones vividas por los pacientes con COVID-19, sin descuidar nunca la participación de los cuidadores.

DESCRIPTORES: Enfermería de rehabilitación; Pandemia; Infecciones por coronavirus

\section{INTRODUÇÃO}

Em dezembro de 2019, é descrita a presença de um novo coronavírus na China, especificamente na cidade de Wuhan, vírus denominado SARS-CoV-2, de elevada transmissibilidade humana e responsável pela doença que ficou designada por COVID-19. Rapidamente, e em poucas semanas, atingiu quase todos os continentes, sendo assumida pela OMS (Organização Mundial da Saúde) como uma pandemia, a 11 de março $2020^{(1-4)}$.

A pandemia surge assim de forma inesperada e envolta de incertezas e indefinições o que gerou insegurança nos comportamentos a adotar, quer pelos profissionais de saúde, quer pela população em geral.

Os serviços de saúde tiveram que se adaptar a uma nova realidade, em particular os dedicados ao tratamento de doenças do foro infecioso e doenças emergentes. No dia 31 de janeiro de 2020 foi admitido, sinalizado pela Direção Geral da Saúde (DGS), o primeiro doente suspeito de COVID-19 em Portugal, num Centro Hospitalar da região Norte do País, num serviço identificado pela DGS com capacidade para receber doentes com patologias emergentes.

A 2 de Março é confirmado o primeiro doente com COVID-19 na mesma unidade hospitalar, umas horas depois do primeiro caso nacional.

Epidemiologicamente, na fase inicial, soube-se que a doença afeta com mais gravidade a população idosa, assim como indivíduos com comorbilidades do foro respiratório, cardiovascular, diabetes Mellitus, insuficiência renal e doença oncológica ${ }^{(5-6)}$. Com as diferentes fases de evolução pandémica, verificou-se que a infeção por SARS-CoV-2 moderada a grave atinge indivíduos mais jovens, saudáveis e sem comorbilidades conhecidas.

Estima-se que cerca de $80 \%$ dos doentes infetados apresente doença ligeira a moderada, contudo 6 a $10 \%$ desenvolvem doença crítica com necessidade de internamento em Unidade de Cuidados Intensivos (UCl). A infeção pode progredir rapidamente para Síndrome de Dificuldade Respiratória Aguda no Adulto ou falência orgânica ${ }^{(3,5,7)}$.

$\mathrm{Na}$ evolução da doença, a pneumonia por SARS-CoV-2, definida como patologia de etiologia restritiva, é a complicação mais frequente, com uma apresentação clínica caraterizada por febre, tosse (seca/produtiva), dor pleurítica, dispneia e fadiga. Sintomas como mialgias, cefaleias, odinofagia, congestão nasal, anosmia e anosgomia, dor articular, shivering, náuseas ou vómitos, diarreia, dor abdominal e hemoptises são também descritos em situação de infeção por SARS-CoV-2 (1-2,4-5,8-9).

Os serviços de saúde tiveram que se adaptar a esta realidade, para garantir em segurança o cuidado aos doentes com esta patologia. As restruturações logísticas e estruturais foram profundas e contínuas, e de acordo com a evolução pandémica. As equipas de profissionais, em particular os enfermeiros, tiveram um papel determinante no 
processo de cuidados durante a pandemia, no planeamento, vigilância, prevenção e controlo da disseminação da doença ${ }^{(10)}$.

Os Enfermeiros Especialistas em Enfermagem de Reabilitação (EEER), como parte integrante das equipas multidisciplinares, tiveram um papel relevante na avaliação e tomada de decisão personalizada sobre o planeamento dos cuidados a prestar aos doentes com COVID-19. Estes profissionais apresentam um conjunto de conhecimentos e competências específicas que lhes permitem maximizar o potencial funcional da pessoa, garantindo as condições necessárias à reconstrução da autonomia e recuperação da independência. A sua atuação promove o diagnóstico precoce e ações preventivas de forma a assegurar as capacidades funcionais da pessoa, prevenir complicações e evitar incapacidades ${ }^{(1,9,10-11)}$.

Decorrente de um processo patológico que afeta frequentemente o sistema respiratório e cujo agravamento terá repercussões em vários sistemas corporais, muitas das necessidades emergentes nos doentes com COVID-19 estão diretamente relacionadas com o core da enfermagem de reabilitação. Todavia, a par do agravamento da condição da pessoa com a doença, o aumento global das necessidades de cuidados dos doentes internados nos serviços e a obrigatoriedade de adotar medidas preventivas que limitassem a transmissão do SARS-CoV-2, impuseram mudanças na forma como os EEER prestavam cuidados especializados.

Neste sentido, conscientes do impacto da COVID-19 nos cuidados especializados em enfermagem de reabilitação que foram prestados aos doentes, ao longo deste contexto pandémico, este estudo tem como objetivo relatar a experiência vivenciada pelos EEER durante a prestação de cuidados especializados num serviço coorte de doentes com COVID-19.

\section{METODOLOGIA}

Este estudo constitui um relato de experiência, descritivo e retrospetivo, referente ao exercício profissional dos enfermeiros especialistas em enfermagem de reabilitação de um serviço coorte de doentes com COVID-19, de um hospital central da região Norte de Portugal, no período de fevereiro de 2020 a julho de 2021. Importa referir que no serviço em causa, ao longo do período a que se refere este relato de experiência, houve sempre internamentos de doentes com COVID-19, embora o número fosse variando.

No que se reporta à Instituição, dados desde o início da pandemia até 11 de julho de 2021, confirmaram que foram identificados 9771 doentes com COVID-19, dos quais 2387 foram tratados em regime de internamento ${ }^{(12)}$.

0 relato de experiência possibilita a aproximação da prática com a teoria. Estudos dessa modalidade descrevem e analisam a aplicação de processos, métodos ou ferramentas, contextualizando a experiência e mostrando os resultados obtidos e lições aprendidas. Neste sentido, este relato de experiência tem como finalidade registar o percurso desenvolvido pelos EEER na área dos cuidados especializados em enfermagem de reabilitação, em contexto de pandemia pela COVID-19, refletindo também nas atividades diagnósticas e nas intervenções de enfermagem mais frequentemente documentadas no SClínico. Está integrado numa investigação mais ampla intitulada "Prática profissional de enfermagem durante a pandemia pela COVID-19: perceções e vivências dos enfermeiros”, autorizada pela comissão de ética em adenda ao Projeto 104-21.

\section{Relato de Experiência}

De fevereiro de 2020 a julho de 2021 , como pessoas e profissionais vivenciamos momentos de incerteza e medo pelo futuro. Porém, o sentido de missão e esperança contribuíram para manter a capacidade de resiliência. 0 fato de se exercer funções num serviço em que a formação em doenças emergentes é contínua, foi uma mais-valia determinante na abordagem dos doentes com COVID-19 e, de uma maneira geral, para enfrentar todo o contexto pandémico. 0 serviço em questão foi referência na reorganização de outros serviços que foram sucessivamente adaptados no âmbito do plano de contingência, para dar resposta à exigência de novos internamentos na instituição.

Especificamente em relação à atuação do EEER é de destacar a pertinência em duas áreas: gestão da equipa e assistência a doentes com COVID-19.

\section{Enfermeiro Especialista em Enfermagem de Reabilitação na Gestão da Equipa}

Os órgãos da gestão de topo da Instituição anteciparam a necessidade de reorganização e estabeleceram vários planos de funcionamento em situação de contingência, com definição sequencial de serviços a abrir em caso de necessidade crescente de internamentos nas diferentes valências de cuidados: nível I/II/III. Das várias medidas implementadas, destacam-se o reforço de formação e divulgação constante de diretrizes emanadas pela DGS, pelos órgãos de gestão da Instituição e Unidade de Prevenção e Controlo de Infeções e de Resistências aos Antimicrobianos (UPCIRA); aquisição de equipamentos de proteção individual (EPI); reforço de material de consumo clínico e hoteleiro, equipamentos de suporte ventilatório para Ventilação Não invasiva (VNI) e para Oxigenoterapia Nasal de 
Alto Fluxo (ONAF) e Capacete de Helmet; bem como contratação de recursos humanos para satisfazer rácios adequados nas coortes.

$\mathrm{Na}$ primeira fase da pandemia, em poucos dias após identificação do primeiro doente com COVID-19, o aumento de casos confirmados foi exponencial. Com a lotação do serviço completa, a nossa realidade e funções enquanto especialistas em enfermagem de reabilitação sofreu alterações, de forma a garantir rácios para manter a qualidade e segurança dos cuidados prestados. No processo de ajuste de equipas foi definida a "figura" de enfermeiro supervisor, elemento estruturante nas equipas multidisciplinares, cuja função é dar apoio a todos os profissionais no âmbito da prestação de cuidados diretos e indiretos, supervisionar o correto cumprimento de todos os procedimentos instituídos, dentro e fora das unidades, bem como, supervisionar a colocação e remoção do Equipamento de Proteção Individual (EPI).

Durante a primeira vaga, os enfermeiros especialistas em enfermagem de reabilitação desempenharam funções de enfermeiro supervisor, mas nunca deixando de olhar para o doente enquanto EEER ajudando toda a equipa multidisciplinar a avaliar, planear, executar e adequar os cuidados às necessidades individuais e únicas de cada pessoa. A título de exemplo: posicionamento terapêutico, gestão de oxigenoterapia, inaloterapia, gestão de suporte ventilatório, mobilizações/transferência de doentes, reconstrução da autonomia e independência nos autocuidados, adequação de suporte nutricional, articulação com família/pessoa significativa e planeamento da alta.

Apesar do contato direto com o doente ser menor, sempre que necessário foi zelada a intervenção direta do EEER. Devido à elevada transmissibilidade do vírus, foi recomendado inicialmente que as intervenções passíveis de gerar aerossolização de gotículas fossem evitadas ${ }^{(1)}$.

Com evolução científica e maior conhecimento sobre o SARS-CoV-2 e a COVID-19, a reabilitação respiratória passou a ser encarada com mais segurança e com outcomes favoráveis nos doentes com esta patologia, nomeadamente no que se refere à redução de dispneia, ao alívio da ansiedade e depressão, à redução de complicações, à prevenção e melhoria da disfunção, diminuição da incapacidade, bem como melhoria da qualidade de vida ${ }^{(1,13)}$.

A gestão de serviço teve sempre presente a relevância do papel do EEER. Para garantir medidas de controlo de infeção e necessidades acrescidas em doentes com necessidades de reabilitação respiratória e motora foi pedido mais material no âmbito da reabilitação: cronómetros, bastões, cicloergómetros, pesos diversos, inspirómetros de incentivo.

\section{Enfermeiro Especialista em Enfermagem de Reabilitação na Prestação de Cuidados}

Embora o conhecimento fosse aumentando ao longo da pandemia, os profissionais de saúde sabiam desde o início que a COVID-19 é uma doença infeciosa respiratória com alto risco de contágio, e que conduz não só à disfunção do sistema respiratório, mas também com repercussões a nível cardíaco, renal, hepático e imunitário ${ }^{(14)}$. Pessoas com COVID-19 com indicação para internamento convencional apresentam frequentemente quadro clínico de insuficiência respiratória tipo I, acompanhado de febre, dispneia, fadiga e dores musculares ${ }^{(15)}$. Atendendo a que muitas dessas áreas de atenção são sensíveis à intervenção dos EEER, os objetivos da sua atuação passam por diminuir a sintomatologia e as complicações decorrentes da imobilidade. Neste sentido, face às recomendações internacionais e às orientações nacionais, é fundamental o investimento dos EEER na cinesiterapia respiratória, assim como no treino de exercício, tendo sempre em consideração a condição clínica das pessoas doentes e o contexto onde os cuidados são prestados ${ }^{(15-16)}$. Convictos do papel fundamental que podiam desempenhar no tratamento e na recuperação dos doentes com COVID-19, os EEER ajustaram a sua intervenção às dificuldades.

Assim, na sequência do menor tempo de contacto com cada doente, os EEER elaboraram flyers (Figuras 1 e 2), com exemplificação de vários exercícios: controlo respiratório, respiração diafragmática, técnica de tosse eficaz e posicionamento de descanso, que eram entregues aos doentes com capacidade de compreensão. De ressalvar que as portas das unidades dos doentes, do serviço em questão, têm um vidro que permite observar os doentes do exterior e todas as enfermarias / quartos tem intercomunicador, o que constituiu uma mais-valia no âmbito do processo de comunicação com os doentes. Sempre que necessário os EEER entravam nas enfermarias e instruíam os exercícios. 


\section{Reabilitação respiratória}

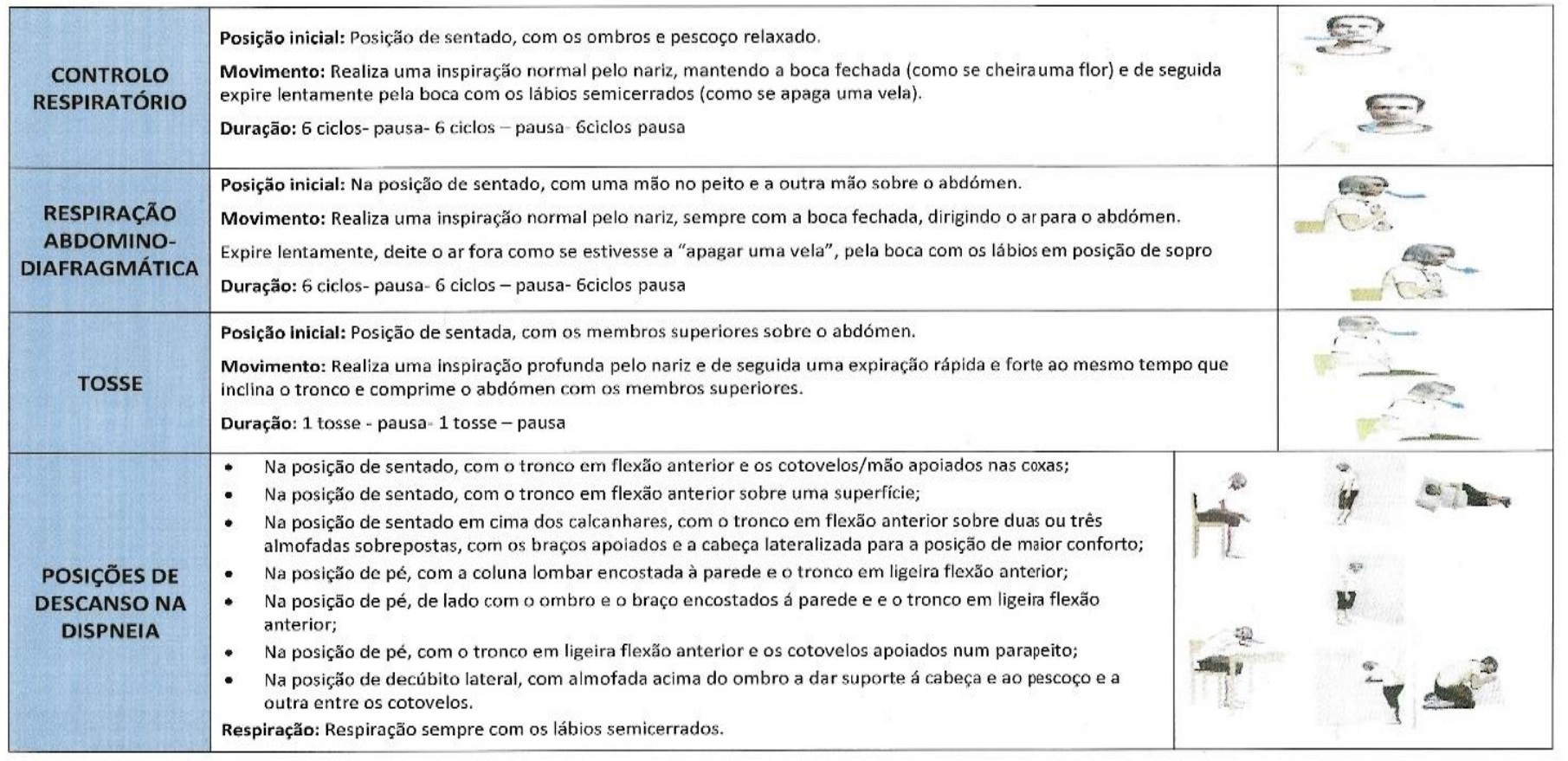

Figura 1 - Flyer de reabilitação respiratória elaborado pelos EEER

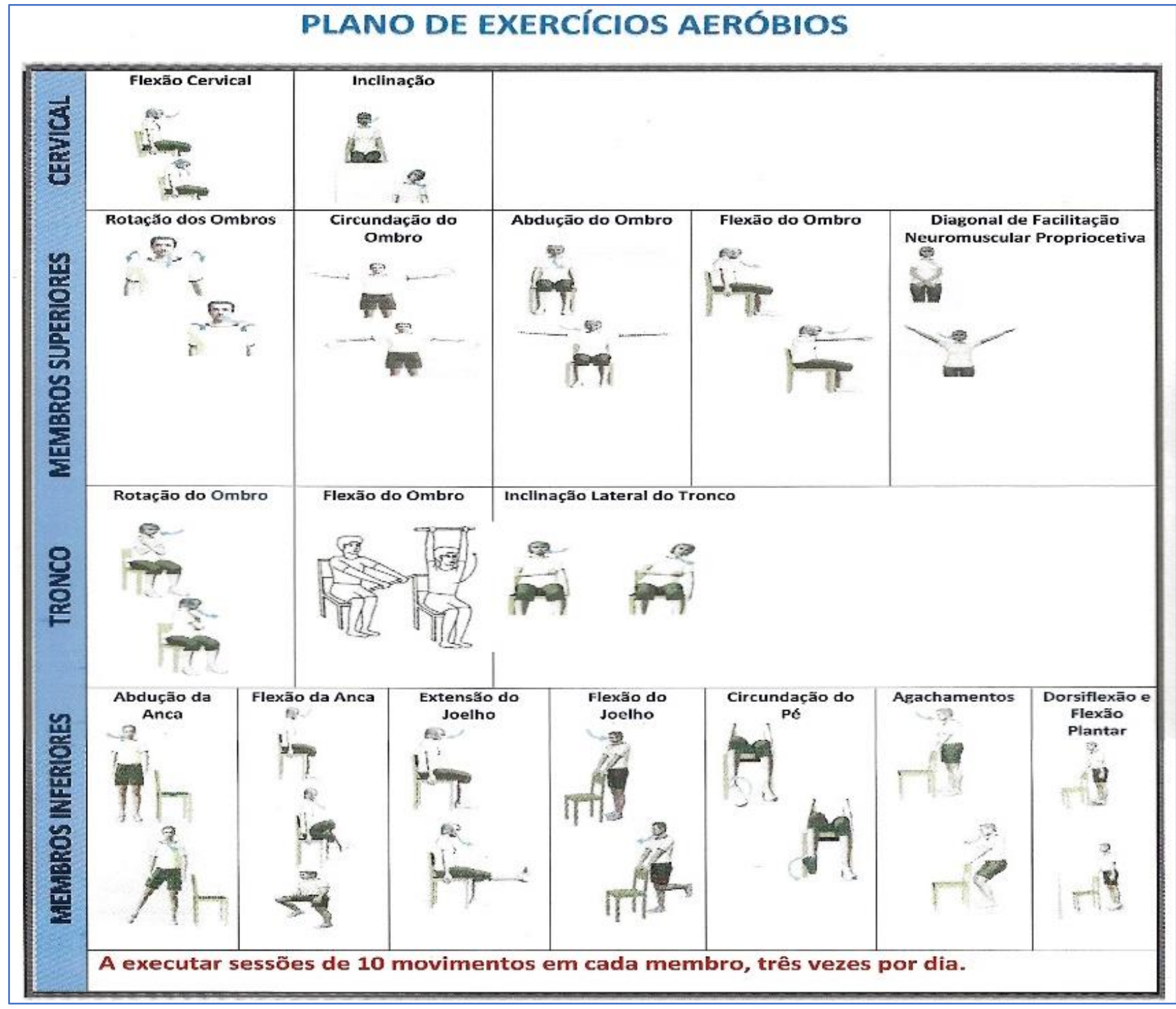

Figura 2 - Flyer de exercícios aeróbios elaborados pelos EEER 
Os primeiros doentes admitidos eram jovens e muitos deles sem critérios major de gravidade, mas que por desconhecimento total da doença ficavam internados aquando do diagnóstico. Com a evolução da pandemia, e inerente ao aumento de casos foram definidos critérios rigorosos de internamento e, naturalmente, a tipologia de doentes alterou.

De março a maio de 2020 o exercício pleno e exclusivo das competências na qualidade de enfermeiro especialista em enfermagem de reabilitação ficou condicionado, no entanto, em junho de 2020 com estabilização de recursos humanos e encerramento de outras coortes, o serviço voltou a ter os EEER dedicados exclusivamente ao exercício das suas competências específicas e sem qualquer tipo de condicionante.

Quando foi possível desempenhar integralmente as funções de EEER, com maior conhecimento científico sobre a doença e maior segurança dos profissionais, verificou-se uma preocupação significativa em manter atualizados no sistema de documentação em uso, os planos de cuidados, o que permitiu não só avaliar os ganhos mas, simultaneamente, perceber mudanças no perfil de registos, nomeadamente no que se refere às atividades diagnósticas (Quadro 1) que permitiram colher dados que sustentaram a identificação de diagnósticos no âmbito da ventilação, do movimento muscular e articular, do equilíbrio e da deglutição, bem como das intervenções mais frequentes (Quadros 2 e 3).

Assim, nos quadros seguintes apresentam-se dados relativos a 2019 (antes da COVID-19), 2020 (com atividade assistencial na área de enfermagem de reabilitação condicionada no período de março a maio) e 2021 (dados de 1 janeiro a 31 de julho de 2021) (17). Importa referir que atendendo à tipologia desta publicação apenas se apresentam nos quadros os registos mais frequentes.

Quadro 1 - Principais atividades diagnósticas documentadas em 2019/2020/2021

\begin{tabular}{|c|c|c|c|}
\hline \multirow{2}{*}{ ATIVIDADES DIAGNÓSTICAS (AD) } & \multicolumn{3}{|c|}{$N^{\circ}$ de $A D$ registadas } \\
\hline & 2019 & 2020 & $2021^{*}$ \\
\hline Avaliar Movimento Muscular & 970 & 994 & 707 \\
\hline Avaliar Capacidade Para Executar Técnicas De Exercício Muscular E Articular & 724 & 655 & 390 \\
\hline Avaliar Capacidade Para Otimizar A Ventilação & 588 & 721 & 590 \\
\hline Avaliar Equilíbrio Corporal & 449 & 463 & 382 \\
\hline Avaliar Deglutição & 195 & 542 & 354 \\
\hline
\end{tabular}

"Os dados de 2021 referem-se ao período de 1 de janeiro até 31 de julho 2021.

Quadro 2 - Intervenções do tipo Executar mais documentadas em 2019/2020/2021

\begin{tabular}{|c|c|}
\hline Intervenções do tipo Executar - 2019 & $\mathrm{~N}^{\circ}$ de intervenções registadas \\
\hline Executar Técnica De Exercitação Musculoarticular Ativa-Resistida & 1499 \\
\hline Executar Técnica De Exercitação Musculo Articular Através De Dispositivos & 1434 \\
\hline Executar Técnica De Reeducação Diafragmática & 1363 \\
\hline Executar Técnica Costal Inferior Bilateral & 1231 \\
\hline Executar Técnica De Exercitação Musculoarticular Ativa-Assistida & 642 \\
\hline Executar Técnica De Exercitação Musculoarticular Passiva & 454 \\
\hline Intervenções do tipo Executar - 2020 & $N^{\circ}$ de intervenções registadas \\
\hline Executar Técnica Costal Inferior Bilateral & 1254 \\
\hline Executar Técnica De Reeducação Diafragmática & 1230 \\
\hline Executar Técnica De Exercitação Musculoarticular Ativa-Resistida & 1133 \\
\hline Executar Técnica De Exercitação Musculo Articular Através De Dispositivos & 1055 \\
\hline Executar Técnica De Exercitação Musculoarticular Ativa-Assistida & 800 \\
\hline Executar Cinesiterapia Respiratória & 309 \\
\hline Intervenções do tipo Executar - 2021 & $\mathrm{~N}^{\circ}$ de intervenções registadas \\
\hline Executar Técnica De Reeducação Diafragmática & 1091 \\
\hline Executar Técnica Costal Inferior Bilateral & 1078 \\
\hline Executar Técnica De Exercitação Musculoarticular Ativa-Resistida & 850 \\
\hline Executar Técnica De Exercitação Musculo Articular Através De Dispositivos & 654 \\
\hline Executar Técnica De Exercitação Musculoarticular Ativa-Assistida & 611 \\
\hline Executar Cinesiterapia Respiratória & 332 \\
\hline
\end{tabular}


Quadro 3 - Intervenções do tipo Ensinar/Instruir e Treinar mais documentadas em 2019/2020/2021

\begin{tabular}{|c|c|}
\hline Intervenções de Enfermagem do tipo Ensinar/Instruir e Treinar - 2019 & $\mathrm{~N}^{\circ}$ de intervenções registadas \\
\hline Ensinar Sobre Exercícios Musculoarticulares & 781 \\
\hline Ensinar Sobre Exercícios Respiratórios & 461 \\
\hline Ensinar Sobre Dispositivos Respiratórios & 446 \\
\hline Ensinar Sobre Ventilação & 431 \\
\hline Ensinar Sobre Exercícios Terapêuticos Para A Deglutição & 29 \\
\hline Instruir A Exercitação Musculoarticular & 866 \\
\hline Instruir Sobre Exercícios Respiratórios & 785 \\
\hline Instruir A Técnica Respiratória & 747 \\
\hline Instruir Sobre Dispositivos Respiratórios & 662 \\
\hline Instruir Sobre Equilíbrio Corporal & 572 \\
\hline Treinar A Técnica De Exercitação Musculoarticular & 973 \\
\hline Treinar A Otimização Da Ventilação Através De Técnica Respiratória & 892 \\
\hline Treinar A Otimizar A Ventilação Através De Dispositivos & 795 \\
\hline Treinar O Equilíbrio Corporal & 605 \\
\hline Treinar A Técnica De Deglutição & 81 \\
\hline Intervenções de Enfermagem do tipo Ensinar/Instruir e Treinar - 2020 & $\mathrm{~N}^{\circ}$ de intervenções registadas \\
\hline Ensinar Sobre Exercícios Musculoarticulares & 1095 \\
\hline Ensinar Sobre Exercícios Respiratórios & 609 \\
\hline Ensinar Sobre Ventilação & 596 \\
\hline Ensinar Sobre Dispositivos Respiratórios & 205 \\
\hline Ensinar Sobre Exercícios Terapêuticos Para A Deglutição & 38 \\
\hline Instruir A Exercitação Musculoarticular & 1057 \\
\hline Instruir A Técnica Respiratória & 1047 \\
\hline Instruir Sobre Exercícios Respiratórios & 1046 \\
\hline Instruir Sobre Equilíbrio Corporal & 750 \\
\hline Instruir Sobre Técnicas Para Aumentar O Equilíbrio Corporal & 463 \\
\hline Treinar A Técnica De Exercitação Musculoarticular & 1186 \\
\hline Treinar A Otimização Da Ventilação Através De Técnica Respiratória & 1121 \\
\hline Treinar O Equilíbrio Corporal & 804 \\
\hline Treinar A Otimizar A Ventilação Através De Dispositivos & 302 \\
\hline Treinar A Técnica De Deglutição & 124 \\
\hline Intervenções de Enfermagem do tipo Ensinar/Instruir e Treinar - 2021 & $\mathrm{~N}^{\circ}$ de intervenções registadas \\
\hline Ensinar Sobre Exercícios Musculoarticulares & 625 \\
\hline Ensinar Sobre Ventilação & 470 \\
\hline Ensinar Sobre Exercícios Respiratórios & 463 \\
\hline Ensinar Sobre Exercícios Terapêuticos Para A Deglutição & 37 \\
\hline Ensinar Sobre Dispositivos Respiratórios & 20 \\
\hline Instruir Sobre Exercícios Respiratórios & 888 \\
\hline Instruir A Técnica Respiratória & 862 \\
\hline Instruir A Exercitação Musculoarticular & 568 \\
\hline Instruir Sobre Equilíbrio Corporal & 380 \\
\hline Instruir Sobre Técnicas Para Aumentar O Equilíbrio Corpora & 589 \\
\hline Treinar A Otimização Da Ventilação Através De Técnica Respiratória & 929 \\
\hline Treinar A Técnica De Exercitação Musculoarticular & 851 \\
\hline Treinar O Equilíbrio Corporal & 435 \\
\hline Treinar A Técnica De Deglutição & 113 \\
\hline Treinar Exercícios Terapêuticos Para A Deglutição & 84 \\
\hline
\end{tabular}


Analisando os dados apresentados, podemos concluir que em 2019 as atividades diagnósticas e as intervenções mais frequentes estavam focadas na reabilitação motora e em 2020, assim como nos 7 primeiros meses de 2021 centraram-se na reabilitação respiratória. Ainda que de março a maio de 2020 , a conceção, a prestação e a documentação dos cuidados de enfermagem de reabilitação estivesse condicionada, é notório o enfoque dos EEER em intervenções com integridade referencial com diagnósticos que emergem do compromisso no sistema respiratório, frequente nos doentes com COVID-19.

Após essa fase inicial, e uma vez garantidas as condições estruturais para que os EEER se dedicassem aos cuidados especializados, a reabilitação respiratória foi contextualizada e adaptada individualmente, tendo em consideração os fatores relacionados com a COVID-19 (fase evolutiva, estabilização/agudização, patologias associadas), com a pessoa (grau de instrução, capacidade de aprendizagem, grau de dependência) e com os recursos disponíveis.

Muitos doentes internados no serviço, na sequência da instabilidade clínica, necessitavam de suporte ventilatório (VNI, ONAF) logo no momento de admissão, e consequentemente, necessidade de cuidados de nível II e III. O facto do serviço em questão ter as valências de cuidados nível I e III, constituiu uma mais-valia para a gestão de cuidados. Nos picos de incidência e, consequentemente, perante o aumento do número de internamentos a nível nacional, houve momentos de grande pressão na gestão de camas e foi muitas vezes necessário gerir cuidados de nível II no internamento. Nestes casos a existência de um sistema de monitorização com telemetria, permitiu garantir a segurança dos cuidados prestados aos doentes, que mesmo numa situação de exigência clínica significativa se encontravam no internamento.

Nestes casos o programa de reabilitação tornava-se difícil de implementar, devido à sintomatologia apresentada pelos doentes. A dispneia, a intolerância à atividade e a tosse constituíam, sem dúvida, a sintomatologia mais incapacitante ${ }^{(9)}$. E na iminência da necessidade de cuidados de nível II e III a intervenção dos EEER centrava-se no controlo sintomático e na otimização da ventilação. Iniciava-se com o doente o processo de reeducação funcional respiratória através de técnicas de relaxamento, consciencialização e controlo respiratório, reeducação diafragmática, posição terapêutica (prone), inspirometria de incentivo e mecanismos de limpeza das vias aéreas. Com o agravamento do estado clínico e a evolução da patologia para pneumonia, a promoção da limpeza das vias aéreas era essencial no processo de tratamento e na recuperação. As técnicas para limpeza das vias aéreas implementadas pelos EEER permitiam mobilizar e eliminar as secreções brônquicas, melhorar a ventilação, promover a reexpansão pulmonar, melhorar a oxigenação e trocas gasosas, diminuir o trabalho respiratório e prevenir complicações. Nos doentes que manifestavam capacidade física e cognitiva, os EEER investiam ainda em intervenções no âmbito do ensinar/instruir/treinar a tosse espontânea e dirigida, ciclo ativo da respiração, pressão expiratória positiva e oscilação torácica. Nos doentes com compromisso na capacidade para se envolver e participar nos cuidados, era mais frequente a técnica de tosse assistida, a drenagem postural, o uso do insuflador/exsuflador mecânico (cough assist) e, em alguns casos, a aspiração de secreções das vias aéreas em circuito fechado.

Com a evolução clínica e consequente estabilização da sintomatologia, o plano e intervenção dos EEER centrava-se na otimização da ventilação, no alívio da sintomatologia respiratória, na diminuição das complicações resultantes da imobilidade, no fortalecimento muscular e treino de exercícios com o objetivo da recuperação funcional, bem como capacitação para o autocuidado, num processo de preparação para a alta e regresso ao domicílio. Em relação a este aspeto, importa referir que os EEER implementaram regularmente intervenções centradas na exercitação muscular e articular dentro das enfermarias, reaproveitando alguns instrumentos já existentes, como por exemplo os degraus da enfermaria para executar treino de subir e descer degraus, de marcha em plano e declive, exercícios ativos-resistidos com pedaleiras, pesos, e treino de autocuidados com o objetivo de reconstruir a autonomia, já que pelo isolamento inerente ao diagnóstico, o treino de subir escadas no serviço e o deambular pelo mesmo deixou de ser opção.

A administração de terapêutica inalatória é parte fundamental no tratamento de patologias respiratórias e a sua correta utilização é importante na prevenção de complicações. Enquanto intervenção interdependente, a inaloterapia foi executada através de câmara expansora, promovendo a diminuição de aerossolização e otimização da técnica à capacidade inspiratória dos doentes. Neste âmbito, de modo a melhorar o conhecimento e a capacitar os doentes e/ou os cuidadores para a técnica inalatória correta, os EEER investiram essencialmente nas intervenções ensinar/instruir/treinar a técnica inalatória aos doentes e/ou aos cuidadores, fornecendo material de leitura (Figura 3) com os passos do procedimento.

Outro dos domínios com destaque na atuação dos EEER refere-se ao equilíbrio e, consequentemente, ao investimento nas intervenções instruir e treinar o equilíbrio, essencial à recuperação dos doentes, bem como à prevenção de complicações. Importa referir que a evolução positiva no equilíbrio, repercutia-se favoravelmente na capacitação para o autocuidado. Avaliar a deglutição foi outra das atividades diagnósticas frequentes, que traduz a preocupação dos EEER em despistarem precocemente alterações na deglutição, nomeadamente casos de disfagia orofaríngea. De facto, a atuação precoce na pessoa com alteração da deglutição (devido à alteração do estado de consciência, diminuição do tónus muscular, tosse ineficaz e aumento das secreções brônquicas) previne complicações, diminui o risco de pneumonia associada à aspiração de conteúdo alimentar, desnutrição e desidratação. A alteração da deglutição é muito prevalente nos doentes que tiveram internamento prévio em unidades de cuidados intensivos $(\mathrm{UCl})$ após ventilação invasiva, o que efetivamente foi frequente nos doentes com COVID-19 internados no serviço. 


\section{TERAPIA INALATÓRIA COM CÂMARA EXPANSORA}

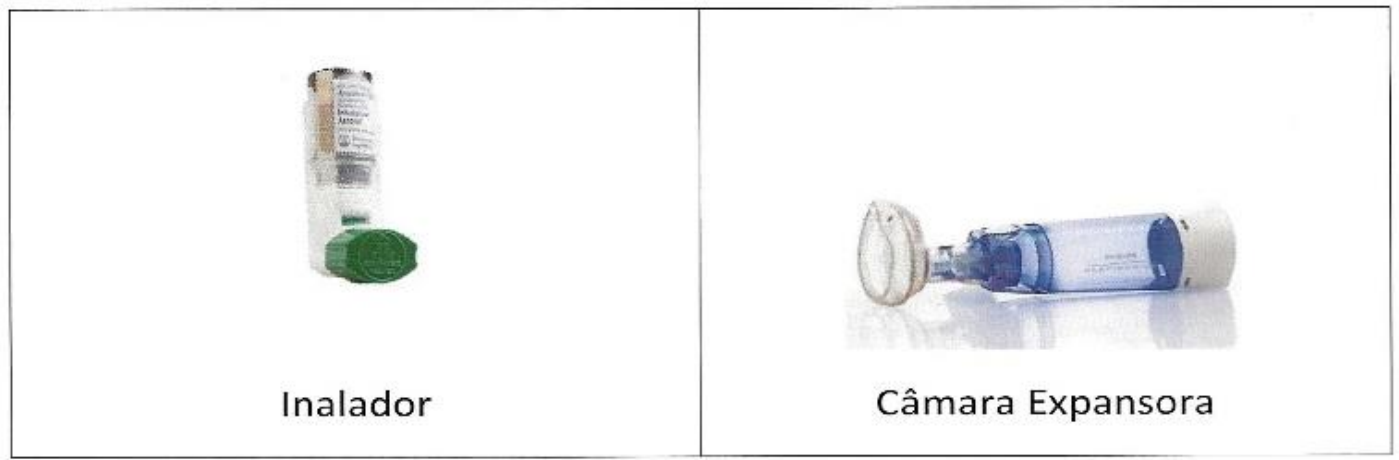

1) Agite o inalador (5 seg);

2) Retire a tampa do bocal;

3) Insira o inalador na peça posterior da câmara expansora;

4) Expire;

5) Aplique a máscara da câmara expansora na sua face; de seguida pressione o inalador no início de uma inspiração lenta;

6) Mantenha a máscara no rosto, inspire e expire através da câmara durante 5-6 respirações.

Nota: Se tiver indicação médica, para realizar uma segunda inalação ( 2 \% puff) deve aguardar 30 a 60 segundos e voltar a agitar o inalador com a tampa fechada recomeçando tudo no passo 1).

Na primeira utilização de cada inalador deverá agitar o inalador e desperdiçar (libertar uma pulverização para o ar) por duas vezes (com intervalo de 10 segundos entre cada pulverização).

Figura 3 - Flyer de Terapia Inalatória com Câmara Expansora elaborado pelos EEER

A gravidade da condição clínica dos doentes com COVID-19 e a complexidade dos cuidados, podem ter determinado a priorização dos EEER em focos de atenção no domínio da função, pelo menos no que se refere à documentação, aspeto que já foi confirmado por outros autores, mesmo antes do contexto pandémico ${ }^{(18)}$.

Em todo este processo, os EEER sentiram limitações no exercício das suas funções. A comunicação enquanto um processo dinâmico, contínuo, através do qual se estabelecem relações e interações humanas e em que é importante valorizar a dimensão verbal e não-verbal, foi significativamente afetada, especificamente, a comunicação terapêutica, fundamental no dia-a-dia do EEER para garantir o sucesso dos procedimentos técnicos, mas essencialmente para promover o envolvimento da pessoa no processo de reabilitação e recuperação.

De ressalvar que o planeamento da alta era estabelecido sempre com envolvimento do familiar/cuidadores, tendo os EEER um papel de dinamizador da integração dos mesmos no planeamento de cuidados. Ainda que em contexto pandémico, tivesse sido instituída a restrição de visitas $(7,11)$, sempre que necessário, além do contacto telefónico, foram criadas as condições para o contacto presencial, já que de outra forma seria difícil garantir a continuidade de cuidados no domicílio. Com o objetivo de manter o programa de reabilitação iniciado no internamento, era elaborado com o doente e cuidador/familiar cuidador um planeamento de reabilitação respiratória e funcional individualizado.

Como condicionantes no processo de reabilitação identificamos o uso do EPI, que dificultava a perceção com voz abafada por duas máscaras, a capacidade auditiva que aparentemente estava diminuída pela presença da cogula e a visão dificultada pela viseira/óculos. Ao referido, acresce o toque alterado pela presença de luvas, a dificuldade em auscultar o doente e a impossibilidade de estabelecer uma comunicação próxima e adequada, o que em alguns casos comprometeu o sucesso da intervenção ${ }^{(1)}$. 


\section{CONCLUSÃO}

$\mathrm{Na}$ fase inicial, a transição do EEER para o papel de supervisor foi justificada pela situação de emergência pandémica, no entanto, estes estiveram sempre presentes no planeamento dos cuidados aos doentes. Neste contexto, foi testada a capacidade de adaptação dos EEER, que nunca perderam o foco e sentido de missão. 0 facto de algumas das intervenções especializadas terem deixado de ser asseguradas diretamente, pode ter comprometido os resultados dos cuidados, retardando a obtenção de ganhos em saúde.

Quando garantidas as condições para os EEER prestarem cuidados especializados verificou-se uma alteração dos focos de atenção. Perante um processo patológico que afeta essencialmente o sistema respiratório é claro o aumento da frequência da atividade diagnóstica: avaliar capacidade para otimizar a ventilação.

Embora durante a fase pandémica seja notória uma diminuição na avaliação da capacidade para executar técnica de exercício muscular e articular, importa referir que essa avaliação nunca foi descurada, na medida em que é essencial na recuperação funcional da pessoa com COVID-19.

Verificou-se também um aumento da atividade diagnóstica avaliar deglutição, o que se justifica pelo facto do compromisso nesse domínio ser prevalente nos doentes críticos com internamento prévio em UCl.

Embora não constitua objetivo deste artigo, decorrente da experiência descrita, ainda que de forma empírica, reconhecemos que a reabilitação respiratória, o posicionamento terapêutico, os exercícios de fortalecimento muscular, as técnicas de adaptação para a concretização das atividades inerentes aos autocuidados, o investimento na adesão ao regime terapêutico e às medidas preventivas, nomeadamente isolamento, contribuíram para reduzir os dias de internamento e preparar o doente e cuidador/familiar cuidador para o regresso a casa em segurança.

\section{REFERÊNCIAS BIBLIOGRÁFICAS}

1 - Raposo P, Mota Sousa LM. Intervenção do enfermeiro especialista em reabilitação na dispneia da pessoa com COVD-19: Relato de caso. Rev Port Enf Reab [Internet]. 2020;3(Sup 2):5-15. Doi: https://doi.org/10.33194/rper.2020.v3.s2.1.5773

2 - Chaves L. 0 impacto da pandemia por covid-19 nos doentes com acidente vascular cerebral: revisão narrativa de literatura. Rev Port Enf Reab [Internet]. 2020;3(Sup 2):29-33. Doi: https://doi.org/10.33194/rper.2020.v3.s2.4.5785

3 - China MFN, Antunes HIB, Martins LMS, Ferreira FAP, Viseu MFJS, Pires MHD. (2020). Cinesiterepia respiratória no doente crítico com CoVID19: A intervenção do enfermeiro de reabilitação - Estudo de caso. Rev Port Enf Reab [Internet]. 2020;3(Sup 2):58-64. Disponível em: https://rper.aper.pt/index.php/rper/article/view/81

4 - Padilha JM, Silva RP. Impacte da pandemia por COVID-19 nos enfermeiros de reabilitação portugueses. Rev Port Enf Reab [Internet]. 2020;3(2):102-107. Doi: https://doi.org/10.33194/rper.2020.v3.s2.15.5842

5 - Mota M, Sousa L, Bico I, Marques MC. Decúbito ventral na síndrome de dificuldade respiratória no adulto após infeção por coronavirus. Rev Port Enf Reab [Internet]. 2020;3(Sup 2):16-22. Doi: https://doi.org/10.33194/rper.2020.v3.s2.8.5796

6 - Almeida JA. Internal Medicine in Centro Hospitalar Universitário S. João and the COVID-19 Pandemic. Med Intensiv. 2020; 1-6. Disponível em: https://portal-chsj.min-saude.pt/uploads/writer_file/document/3358/Artigo_de_Opini_o.pdf

7 - Cardoso MFPT, Martins MMFPS, Trindade LL, Ribeiro OMPL, Fonseca EF. A pandemia por COVID-19 e as atitudes dos enfermeiros frente à morte. Rev. Latino-Am. Enfermagem [Internet]. 2021;29:e3448. Doi: https://doi.org/10.1590/1518.8345.4769.3448

8 - Silva L, Mota A, Sousa L. Efeito de um programa de (tele)reabilitação respiratória na pessoa com COVD-19: Um estudo de caso. Rev Port Enf Reab [Internet]. 2020;3(Sup 2):23-8. Doi: https://doi.org/10.33194/rper.2020.v3.s2.3.5783

9 - Neto AR, Carvalho ARB, Oliveira EMN, Magalhães RLB, Moura MEB, Freitas DRJ. Symptomatic manifestations of the disease caused by coronavirus (COVID-19) in adults: systematic review. Rev Gaucha Enferm [Internet]. 2021;42(spe):e20200205. Doi: https://doi.org/10.1590/1983-1447.2021.20200205.

10 - Portugal. Regulamento n. ${ }^{\circ}$ 392/2019, de 03 de maio de 2019. Regulamento das competências específicas do enfermeiro especialista em Enfermagem de Reabilitação. Diário da República, 2. ${ }^{a}$ série 3 mai 2019; (8): 13565-13568.

11 - Cardoso MF, Martins MM, Ribeiro OM, Fonseca EF, Pereira VL. Enfermeiros de reabilitação e as atitudes face à morte em contexto de crise pandémica por COVID-19. Rev Port Enf Reab [Internet]. 2020;3(Sup 2):42-49. Doi: https://doi.org/10.33194/rper.2020.v3.s2.6.5792

12 - Portal de Dados, Estatísticas do Internamento; 2020/2021.

13 - Rocha B. O Papel do enfermeiro de reabilitação e a pandemia COVID-19 [Internet]; 2020. Disponível em: https://www.aper.pt/Ficheiros/Covid19/0\%20PAPEL\%20DO\%20ENFERMEIRO\%20DE\%20REABILITA\%C3\%87\%C3\%830\%20E\%20A\%20PANDEMIA\%20C OVID-19.pdf.

14 - World Health Organization. Clinical management of severe acute respiratory infection (SARI) when COVID-19 disease is suspected. [Internet]; Genebra: World Health Organization; 2020 [cited 2022 Jan 04]. Disponível em: https: / / apps. who.int/iris/bitstream/handle/10665/331446/WHO-2019-nCoV clinical-2020.4-eng.pdf?sequence=1\&isAllowed=y

15 - Ordem dos Enfermeiros. ORIENTAÇÕES - COVID-19. Cuidados de Enfermagem de Reabilitação para pessoas com COVID-19. [Internet]; Lisboa: Ordem dos Enfermeiros; 2020 [cited 2022 Jan 04]. Disponível em: https://www.ordemenfermeiros.pt/media/17940/mesa-do-col\%C3\%A9gioda-especialidade-de-enfermagem-de-reabilita\%C3\%A7\%C3\%A3o-orienta\%C3\%A7\%C3\%B5es-covid-19.pdf

16 - Zhao HM, Xie YX, Wang C. Chinese Association of Rehabilitation Medicine; Respiratory Rehabilitation Committee of Chinese Association of Rehabilitation Medicine; Cardiopulmonary Rehabilitation Group of Chinese Society of Physical Medicine and Rehabilitation. Recommendations for respiratory rehabilitation in adults with coronavirus disease 2019. Chin Med J [Internet]. 2020;133(13):1595-1602. https: //doi.org/10.1097/CM9.0000000000000848

17 - Portal de Dados, Sclínico do Internamento; 2020/2021.

18 - Ribeiro OMPL, Martins MMFPS, Tronchin DMR, Forte ECN. Aplicação do processo de enfermagem em hospitais portugueses. Rev Gaúcha Enferm [Internet]. 2018;39:e2017-0174. Doi: https://doi.org/10.1590/1983- 1447.2018.2017-0174. 


\section{DIVULGAÇÕES ÉTICAS}

Contribuição do(s) autor(es):

Conceptualização: MAL; MT; SP; OR

Metodologia: MT; OR;

Validação: MAL; MT; SP; OR;

Análise formal: MAL; MT; SP; OR;

Investigação: MAL; MT; SP; OR;

Tratamento de dados: MAL; MT; SP; OR;

Preparação do rascunho original: MAL; MT; SP; OR

Redação e edição: MAL; MT; SP; OR;

Revisão: MAL; MT; SP; OR

Todos os autores leram e concordaram com a versão publicada do manuscrito.

\section{Financiamento:}

Este trabalho não recebeu nenhuma contribuição financeira ou bolsa.

\section{Comissão de Ética:}

Estudo integrado numa investigação mais ampla intitulada "Prática profissional de enfermagem durante a pandemia pela COVID-19: perceções e vivências dos enfermeiros", autorizada pela comissão de ética em adenda ao Projeto 104-21.

\section{Conflitos de interesse:}

Os autores não declaram nenhum conflito de interesses.

Proveniência e revisão por pares:

Não comissionado; revisto externamente por pares.

(c) Autor (es) (ou seu (s) empregador (es)) e APER/RPER 2022. Reutilização permitida de acordo com CC BY-NC-ND. Nenhuma reutilização comercial. 